\title{
Meaningless Questions in Cosmology and Relativistic Astrophysics
}

\author{
M. Heller \\ Obserwatorium Astronomiczne Uniwersytetu Jagiellońskiego, Kraków, Poland \\ and M. Reinhardt \\ Astronomisches Institut der Ruhr-Universität, Bochum, FRG
}

(Z. Naturforsch. 31 a, 1271-1276 [1976] ; received June 26, 1976)

\begin{abstract}
After a general classification of meaningless questions in science we concentrate on empirically meaningless questions. Introducing the concepts of informationally connected, semiconnected and disconnected observers, a formalism for the analysis of the informational structure of space-time is developed. We discuss some problems of epistemological nature in cosmology and black hole physics. A number of questions like "What was 'before' the initial singularity of the universe?" or "What is the fate of matter in gravitational collapse inside the event horizon?" turn out to be empirically meaningless. We also show that a "wormhole" does not violate causality for the set of informationally connected observers who do not enter it.
\end{abstract}

\section{Introduction}

Questions of epistemological nature often occur in cosmology and in relativistic astrophysics, in particular after the boom in black hole physics. Frequently these problems are either treated in a sloppy way, or their epistemological nature is not even recognized. In the present paper we attempt to sort out physically meaningless questions in this field.

In philosophy the term "meaningless" is historically burdened with the definition of "meaning" by the positivistic and neopositivistic schools of thought. The Vienna circle called a statement, even a universal one, "meaningless", if it is not empirically "verifiable" 1-4. They identified "non-empirical" and "non-verifiable" with "meaningless" and "metaphysical". This definition was so narrow that it excluded physical laws, which are always universal statements, from the realm of science. Popper $^{5}$ sharpened the "criterion of demarcation" between science and non-science by substituting the term "verifiability" by "falsifiability" of a statement. This was an improvement with respect to the problem of empirical testability of scientific theories.

However even Popper's approach to the testability of theories has been criticized as still too narrow ${ }^{6,7}$. We will not deal here with the complicated problem of the relations between theories and empirical data. Our scope is much simpler. We are

Reprint requests to M. Reinhardt, Astronomisches Institut der Ruhr-Universität, Postfach 102148, D-4630 Bochum, FRG. going to define and discuss meaningless questions in relativistic astrophysics and cosmology. In general we call a question meaningless, if there is in principle no answer to it. Since there are different kind of possible answers, we distinguish several classes of meaningless questions.

Meaningless questions of the first kind are of tautological nature. These are meaningless questions by definition. If, for example, we define the universe as "everything that exists", the question "what is outside the universe?" is tautologically meaningless.

Meaningless questions of the second kind are theory dependent. These are questions to which one cannot get an answer within the framework of a given theory. There are two subclasses: theoretically and empirically meaningless questions. A question is theoretically meaningless in the framework of a given theory, if the theory does not provide any tools to answer the question. E. g. questions about quantum effects are theoretically meaningless in a classical field theory like Maxwell's theory. Questions are empirically meaningless, if in principle there cannot be an empirical (observational) answer to them within the framework of a given theory. They relate to empirically untestable predictions of a theory and do occur often in black hole physics (see Section IV).

In a given theory a theoretically meaningless question is also empirically meaningless, since the theory is unable to give any answer to the question, which could be tested empirically. The reverse is not true. A question may be empirically meaningless and yet theoretically meaningful. This situation does arise in relativistic astrophysics. General relativity 
is quite capable to make predictions about regions of space-time which will never be observable to us in the framework of the theory (see Sects. III and IV). Although we will never be able to check these predictions of the theory empirically, their mathematical treatment can help considerably towards a better understanding of the theory. In the following we will concentrate on the problem of empirical meaning.

If one asks a question by setting up an observational device, to which one cannot obtain, in principle, an answer in terms of measurable data, i.e. any information, this question is empirically meaningless. Even if the question is theoretically not meaningless, it can never be used to test the theory. Thus the central term of scientific epistemology is information.

\section{Informational Structure of Space-Time}

We consider space-time, i. e. the set $\mathcal{M}=$ $\{p, q, \ldots, x, y, \ldots\}$ of elements called events, and two subsets of $\mathcal{M}: \mathcal{L}=\left\{L_{1}, L_{2}, \ldots\right\}$, the elements of which are called light rays, light signals or simply photons, and $\mathcal{P}=\{P, Q, \ldots, X, Y, \ldots\}$ the elements of which are called observers. Note that the words "photon", "observer" etc. are used (for brevity) instead of "history (or world line) of a photon or observer" 8 . In principle every timelike curve may be considered as an observer, however in the following we shall consider only geodesic observers, i. e. timelike geodesics.

If from an observer $P$ a light signal is emitted towards an observer $Q$, reflected at $Q$ and received back in a finite proper time at $P$, we call it an echo ${ }^{7}$ from $Q$ to $P$ and we write $P|>| Q$.

If there is an echo from $Q$ to $P$ we say that $P$ is informationally connected with $Q$. We express this more precisely as follows.

\section{Definition 1:}

$P$ is informationally connected with $Q$, with respect to $U \subset \mathcal{M}, P|\underset{U}{>}| Q$, iff for every $p_{1} \in P \cap U$ there are a $q \in Q$ and a $p_{2} \in P \cap U$ such that $p_{1} \unlhd q$ and $q \unlhd p_{2}$, with the condition that $\left|t\left(p_{2}\right)-t\left(p_{1}\right)\right|$ is finite.

Here $\left|t\left(p_{2}\right)-t\left(p_{1}\right)\right|$ denotes the proper time of the observer $P$ elapsed between the events $p_{1}$ and $p_{2}$. $p \unlhd q$ is the so-called horizmodial relation; it should be read " $q$ lies on the light cone of $p$ " 9 . Proper time is measured by atomic, thermodynamical, or biological clocks, which can be non-metric and irregular. The only requirement is that these clocks are monotonical, i.e. that informations arriving continuously to an observer form an ordered set ${ }^{8}$.

If a light signal emitted by an observer $Q$ is received by an observer $P$ we call it a message 8 from $Q$ to $P$. If there is a message from $Q$ to $P$ we say that $P$ is informationally semiconnected with $Q$, or more precisely:

\section{Definition 2:}

$P$ is informationally semiconnected with $Q$, with respect to $U \subset \mathcal{M}, P \bigvee_{U} Q$, iff for every $p \in P \cap U$ there is a $q \in Q$ such that $q \unlhd p$.

Obviously we have: $P|\underset{U}{>}| Q \Rightarrow P \bigvee_{U} Q$.

\section{Definition 3:}

If $P|>| X \Rightarrow \exists V \in \mathcal{M}$ such that $Q \mid \underset{V}{>} P$, we call $U$ and $V$ informationally symmetric.

\section{Definition 4:}

If $P \underset{U}{>}|Q \Rightarrow Q| \underset{U}{>_{U}} P$, we call $U$ informationally normal. Every informationally normal set is informationally symmetric with itself. Minkowski flat space-time is informationally normal.

On on informationally normal set we have:

$$
P|>| Q \Leftrightarrow Q \wedge P \wedge P \wedge Q
$$

We define the set of informationally connected observers to the observer $P$ with respect to $U \subset \mathcal{M}$ :

\section{Definition 5:}

$$
\operatorname{SIC}_{U}(P) \equiv\{X \in \mathcal{P}: P|>| X\}
$$

A $\operatorname{SIC}_{V}(P)$ is called an extension of $\operatorname{SIC}_{U}(P)$, if $U \subset V$ and $\operatorname{SIC}_{U}(P) \subset \mathrm{SIC}_{V}(P)$. A set of informationally connected observers is called inextendible, if $\forall V \supset U, V \subset \mathcal{M}: \operatorname{SIC}_{U}(P)=\operatorname{SIC}_{V}(P)$. We define the set of informationally semiconnected observers to the observer $P$ with respect to $U \subset \mathcal{M}$ :

\section{Definition 6:}

$$
\operatorname{SIS}_{U}(P) \equiv\left\{X \in \mathcal{P}: P \bigvee_{U} X\right\}
$$

Inextendible sets of informationally semiconnected observers are defined as above with SIS instead of SIC everywhere. 


\section{Proposition 1:}

Let $N$ be an informationally normal region of space-time. Every observer in $N$ belongs to exactly one of pairwise disjoint sets of informationally (semi) connected observers.

Proof. Within $N,|>|$ and $N$ are equivalence relations. (Attention: relations $|>|$ and $N$ are defined relatively to a subset $U$ of $\mathcal{M}$ !)

For abstractions of these relations we may simply write $\mathrm{SIC}_{i}, \mathrm{SIS}_{i}(i=1,2, \ldots)$ without reference to a single observer.

\section{Proposition 2:}

$$
\operatorname{SIC}_{U}(P) \cup \operatorname{SIS}_{V}(P)=\operatorname{SIS}_{U} \cup{ }_{V}(P) .
$$

The set of observers may have a more complicated structure, for instance we may consider an informationally semi-connected set of informationally connected observers defined by:

$$
\begin{gathered}
(\mathrm{SIC})_{1} \backslash(\mathrm{SIC})_{2} \text { i. e. for every } \\
P \in(\mathrm{SIC})_{1} \exists Q \in(\mathrm{SIC})_{2} \text { such that } P \backslash Q .
\end{gathered}
$$

\section{Definition 7 :}

An observer $Q$ is informationally disconnected with $P$ with respect to $U$, iff $Q \notin \operatorname{SIS}_{U}(P)$. For this we write $P \| Q$.

\section{Definition 8:}

An observer $Q$ is informationally disconnected with $P$, iff $Q \notin \operatorname{SIS}_{U}(P)$, with $\operatorname{SIS}_{v}(P)$ inextendible, $P \| Q$.

For a given observer $P$ a question is empirically meaningless, if it relates to observers informationally disconnected with $P$.

If a question is empirically meaningless to $P$, it is also empirically meaningless to all observers in the inextendible $\operatorname{SIC}_{U}(P)$.

Let us consider for example two sets of informationally connected observers $\mathrm{SIC}_{1}$ and $\mathrm{SIC}_{2}$, such that $\mathrm{SIC}_{2} \backslash \mathrm{SIC}_{1}$ and $\mathrm{SIC}_{1}|| \mathrm{SIC}_{2}$. This means that $\mathrm{SIC}_{2}$ can receive information from $\mathrm{SIC}_{1}$. On the other hand $\mathrm{SIC}_{1}$ can never receive any information from any of the elements of $\mathrm{SIC}_{2}$. This implies that it is empirically meaningful for an observer, who is an element of $\mathrm{SIC}_{2}$, to ask questions about events in $\mathrm{SIC}_{1}$, but not vice versa. In other words $\mathrm{SIC}_{1}$ and $\mathrm{SIC}_{2}$ are divided by an informationally semipermeable membrane. Examples of informationally semi-permeable membrans are the event horizons in the Schwarzschild and Kerr solutions of general relativity.
One can construct as a "gedankenexperiment" multiply semiconnected sets of observers $\mathrm{SIC}_{i}(i=$ $1, \ldots, n)$ so that some $\mathrm{SIC}_{k}$ can receive informations from $\mathrm{SIC}_{l}$ to $\mathrm{SIC}_{l+m^{\prime}}$ which are all mutually informationally disconnected, but not vice versa, or so that $\mathrm{SIC}_{2}$ to $\mathrm{SIC}_{n}$, all mutually informationally disconnected, can receive informations from $\mathrm{SIC}_{1}$, but not vice versa (e.g. $n-1$ black holes in the universe $\mathrm{SIC}_{1}$ ). One can image also a hierarchy of semiconnected sets, so that $\mathrm{SIC}_{i}$ can transmit information to $\mathrm{SIC}_{i+1^{\prime}}$ but not the other way round. Obviously by combination of these possibilities one can construct very complicated informational structures. We will not go into further details of this informational topology. The main point is that, however complicated the structure for any given SIC, it is uniquely determined what empirically meaningful and meaningless questions are. Thus the notion "empirically meaningless question" is relative, but well defined for any given SIC, if the informational structure is known.

\section{Meaningless Questions in Cosmology}

Let us first define the universe.

\section{Definition 9:}

The universe is the maximum extension of the set of informationally connected observers to which we belong $\left[\mathrm{SIC}_{U}(\mathrm{We})\right.$ inextendible $]$.

"We" does not necessarily mean mankind or the earth. "We" can be generalized to signify a suitable informationally connected subset like our Galaxy, the Local Group, or even the Local Supercluster. An alternative definition of the universe is

\section{Definition $9^{\prime}$ :}

The universe is the set of all observers that can ever transmit information to us $\left[\mathrm{SIS}_{U}(\mathrm{We})\right.$, where $U$ denotes the proper past].

It is worth to emphasize that even in an informationally normal space-time and even if $U=\mathcal{M}$ the universe according to def. 9 is not the entire space-time [see proposition 1 , and note in def. 1 the condition: $\left|t\left(p_{2}\right)-t\left(p_{1}\right)\right|$ is finite].

Note that the two definitions are not identical. Definition $9^{\prime}$ is weaker than def. 9, for in principle we could be an element of $\mathrm{SIC}_{2}\left(\mathrm{SIC}_{2} \wedge / \mathrm{SIC}_{1}\right.$, $\mathrm{SIC}_{1} \| \mathrm{SIC}_{2}$ ), or an element of an informationally more complicated structure. In this case we might 
receive information from observers who are not elements of the maximum extension of the SIC to which we belong. On the other hand def. 9 and $9^{\prime}$ would coincide if we were in $\mathrm{SIC}_{1}$ of a simply or multiply informationally semiconnected set of observers - $\mathrm{SIC}_{1}$ being inextendible and the informational structure being such that no information from other SIC can get to it. Note that this is true if general relativity holds.

Let us consider the following questions:

(i) What is outside the universe?

(ii) What was before the beginning of the universe?

(iii) What will be after the end of the universe?

On the first glance and intuitively they seem to be meaningless questions of the first kind, i.e. tautologically meaningless. In the light of chapter II things are more complicated.

According to def. $9^{\prime}$ these questions are empirically meaningless, since no observer, who is outside the universe, or who existed before the beginning, or will exist after the end of the universe could transmit information to us. This is trivial, for according to def. $9^{\prime}$ an observer who can transmit information to us must be part of the universe. On the other hand, according to def. 9, question (i) might not be empirically meaningless, if we were e. g. an element of $\mathrm{SIC}_{2}$ of an informationally semiconnected set of observers. In this case questions (ii) and (iii) would also be meaningful, if the informationally semipermeable membrane between $\mathrm{SIC}_{1}$ and $\mathrm{SIC}_{2}$ come into existence in the finite past of the observers who are now elements of $\mathrm{SIC}_{2}$. Of course these considerations are of a rather academic nature, since it is hardly possible to find out whether two observers are informationally connected or semiconnected, if the exchange of informations would take several billion years of proper time. Question (ii) needs some discussion, since there might be some misunderstanding of the term "beginning". At present it is generally accepted that the universe was in a very dense and hot stage some $10^{10}$ a ago. General relativity predicts a singularity as the starting point. The singularity would have been a state of infinite density (and infinite temperature, see however ${ }^{10,11}$ ). It has been repeatedly discussed what preceded this singularity (see e. g. ${ }^{12,13}$ ). If general relativity holds up to infinite densities (which is open to doubt), i. e. if the expansion of the universe started with a true singularity, no signals can have reached us from the previous state ${ }^{*}$, if there ever was one, nor will be any transmitted to the next state after the collapse of the universe to a second singularity, if the universe is closed (which seems unlikely at present ${ }^{14}$ ). Thus if general relativity does not break down at some finite density, it is empirically meaningless to ask about states of the universe before the big bang, or after a possible recollapse. On the other hand, if general relativity cannot be extrapolated to infinite densities, i. e. if the universe did not start from a singularity, signals from the state of the universe before the big bang could have been transmitted to us. These difficulties are apparently theory dependent. It is not impossible (and even desirable) that a future theory will overcome all of them.

In this case it would make sense to ask questions about the state of the universe before the big bang and after a possible recollapse to extreme densities. But, of course, then the big bang would not be epistemologically the beginning of the universe.

Question (ii) is closely related to the problem of the initial conditions of the universe. Zel'dovich and Novikov ${ }^{12}$ have shown that in a bouncing closed Friedmann universe information will be transmitted to the next state through the singularity in form of initial conditions, e.g. the entropy per baryon. However, if the big bang started with a true singularity, no signals, i.e. no direct informations, could have crossed it, and we can never check whether the initial conditions are the consequence of previous cycles of a bouncing universe, or whether there were no previous cycles and the universe started with the initial conditions as they were, without any further "explanation". Thus, if general relativity holds strictly, all questions concerning the origin of initial conditions are empirically meaningless, since we cannot receive signals from beyond the singularity.

There are attempts to connect the initial conditions of the universe with the laws of physics (see e. g. ${ }^{15}$ ). Let us suppose that this program would be successful, i. e. that one could prove a one to one correspondence between physical laws and the initial conditions of the universe. Certainly this would be a progress to some extent. However, it would

\footnotetext{
* Information in the form of initial conditions can be transmitted through the singularity ${ }^{12}$. See however the discusion below.
} 
only show that the laws of physics are as they are because the initial conditions of the universe were as they were, or vice versa. It would not help a iota towards the problem of the origin of the initial conditions of the universe. The question, what preceeded the initial singularity would remain empirically meaningless.

\section{Meaningless Questions in Black Hole Physics}

In the last section we have touched upon meaningless questions of the second kind. This section deals wholly with theory dependent meaningless questions under the assumption that general relativity is valid. There were repeated attempts to discuss the physics within the Schwarzschild radius (see e.g. ${ }^{13,16}$ ). We are going to show that it is empirically meaningless to ask questions about the physics inside an event horizon for observers who are outside. We will also show that the existence of "wormholes" can never be proven.

Let us first deal with ordinary gravitational collapse. Suppose there is a black hole surrounded by a $\mathrm{SIC}_{1}$. It does not matter whether the black hole was there from the beginning of the universe, or whether it is in the process of formation. Suppose further that an element of this $\mathrm{SIC}_{1}$ (a sort of kamikaze pilot) descends into the black hole. Because of the time dilatation he will never reach the event horizon of the black hole within finite proper time of any element of the SIC who has stayed safely outside. Of course the kamikaze observer will cross the event horizon, which is in the simplest case the Schwarzschild radius, in a finite interval of his proper time. However, this does not matter to the rest of $\mathrm{SIC}_{1}$ outside. Since no signal can ever cross the event horizon from inside in gravitational collapse (we will discuss the reversed gravitational collapse later) the observers inside the event horizon $\left(\mathrm{SIC}_{2}\right)$ and the observers outside $\left(\mathrm{SIC}_{1}\right)$ form a semiconnected set of observers. (In the language of Section II: $\mathrm{SIC}_{2} \backslash \mid \mathrm{SIC}_{1}, \mathrm{SIC}_{1} \| \mathrm{SIC}_{2}$.)

The kamikaze observer - assume for the moment that he is not torn to pieces by tidal forces - will know within finite proper time what physics is like inside the event horizon, but he will never be able to tell his former fellow observers outside. For them, he will never even reach the event horizon, he will always remain, though increasingly dimly, a member of the original $\mathrm{SIC}_{1}$. The guy who has decided to commit suicide by plunging into a black hole will never disappear completely for his fellows of $\mathrm{SIC}_{1}$. For him it will be a quick and merciful death. For them it will look like an infinitely prolonged, but never completed suicide.

Thus, jokes asides, it is empirically meaningless to ask about the fate of matter once it crosses the event horizon of a singularity - which it never does for the $\mathrm{SIC}_{1}$ outside - or about the physics inside the event horizon, since no signal can ever cross it from inside in gravitational collapse.

Now let us consider the "wormhole" hypothesis, which was brought up by Wheeler ${ }^{17}$. In Kruskal coordinates the Schwarzschild solution consist of two timelike and two spacelike regions. The idea of the "wormhole" is to identify the asymptotically flat timelike regions $\left(\mathrm{R}^{+}\right.$and $\left.\mathrm{R}^{-}\right)$, so that a gravitational collapse occuring in $\mathrm{R}^{+}$would come out as a reversed gravitational collapse in $\mathrm{R}^{-}$, i. e. in the same space-time, but at another place and time. It has been claimed that "wormholes" of this kind would violate causality ${ }^{13}$. One can easily show that for the observers who stay outside - and only they matter epistemologically - causality is not violated. First of all, an observer falling into a black hole has to cross another, "inner" timelike region before he comes out through a white hole in another, or the same universe ${ }^{12}$. In this intermediate region all information from his previous life is erased ${ }^{12}$. But even if this were not so, all questions about "wormholes" are empirically meaningless for the observers outside (i. e. in $\mathrm{R}^{+}$and $\mathrm{R}^{-}$, even if they are identified). Suppose there is a kamikaze observer, element of a $\mathrm{SIC}_{1}$, who tries to get into a "wormhole". In his own proper time he will be able to cross the first event horizon, to pass through the "wormhole" and come back through the second event horizon. Then he will see himself plunge into the black hole end of the wormhole. For him causality would be violated. However this does not matter. He will never be able to tell anybody. The observers, who are the elements of SIC $_{1}$ which have stayed at a safe distance to the black hole (or "wormhole"-nobody in $\mathrm{SIC}_{1}$ will ever know) will never even see their suicidal fellow disappear, and thus of course not reappear at another point of space-time. Thus for the $\mathrm{SIC}_{1}$ outside the "wormhole" causality cannot be violated by this procedure. If "white holes" would exist, i.e. if we could observe the reversed gravitational collapse, questions about the physics 
inside the Schwarzschild radius would not be empirically meaningless, since the $\mathrm{SIC}_{1}$ outside would receive information from this region. However the existence of "white holes", i. e. of regions of spacetime which started to expand later than the rest of the universe ${ }^{12}$, is highly improbable because of quantum effects in strong gravitational fields which limit the retardation to minute fractions of a second.

\section{Final Remarks}

In science there is no place for tautologically meaningless questions, they are completely empty and useless. Questions like "What 'preceded' the initial singularity?" or "What happens to matter in gravitational collapse beyond the event horizon?" are often considered as deep and fundamental issues in physics. We have shown that according to our definition they are empirically meaningless because they relate to predictions of a theory on which in the framework of this theory no empirical data will ever be available. We do not say that research on

1 H. Reichenbach, The Rise of Scientific Philosophy, University of California Press, Berkeley 1951.

2 L. Wittgenstein, Tractatus Logico - Philosophicus, Cambridge Univ. Press, Cambridge 1922.

3 M. Schlick, Naturwiss. 19, 150 [1931].

${ }^{4}$ F. Waismann, Erkenntnis 1, 229 [1930].

5 K. Popper, The Logic of Scientific Discovery, Hutchinson, London 1959.

6 V. S. Shvyrev, in Sovremennye problemy teorii poznania dialekticheskogo materialsma, Ed. M. B. Mitin, Mysl, Moscow 1970, Chapter 4.

7 W. Van Orman Quine, From a Logical Point of View, Harvard University Press, Cambridge 1964.

8 J. Ehlers, F. A. E. Pirani, and A. Schild, in General Relativity, Ed. L. O'Raifertaigh, Clarendon Press, Oxford 1972, p. 63.

9 B. Carter, General Relativity and Gravitation 1, 349 [1971]. this questions is futile. If a question is theoretically meaningless, perhaps it would stimulate the development of a new theory. If a question is experimentally meaningless, it might help us towards a better understanding of the present theory. In this respect our terminology is different from that commonly accepted in the philosophy of science, which has reserved the term "meaningful" only for experimentally meaningful statements. We believe, however, that our understanding of the problem is not far from common practice in science and common understanding in the philosophy of science. We strongly stress the scientific role of empirically meaningful questions. Even if a theory passes all tests, past and future, in the maximum extension of a set of informationally (semi) connected observers, the predictions of this theory about observers (or events), who can never transmit information to any element of it, are untestable (unfalsiable) and thus experimentally meaningless. In other words, one may ask these questions (providing they are not tautologically meaningless) but they cannot be checked by observations or measurements.

10 R. Hagedorn, Cargése Lectures in Physics, VI, Ed. E. Schatzman, Gordon and Breach, New York 1973.

11 A. D. Sakharov, Zhurn. Eks. Teor. Fiz. Pis'ma 3, 349 [1966].

12 Ya. B. Zel'dovich and I. D. Novikov, Relativistskaya Astrofizika, Nauka, Moscow 1967.

13 C. W. Misner, K. S. Thorne, and J. A. Wheeler, Gravitation, Freeman, San Francisco 1973.

14 J. R. Gott III, J. E. Gunn, D. N. Schramm, and B. M. Tinsley, Astrophys. J. 194, 543 [1973].

15 W. Kundt, Springer Tracts in Physics 49, 1971.

16 M. J. Rees, R. Ruffini, and J. A. Wheeler, Black Holes, Gravitational Waves and Cosmology, Gordon and Breach, New York 1975, Chapter 19.

17 J. A. Wheeler, Geometrodynamics, Academic Press, New York 1962. 